\title{
ORIGINAL
}

\section{CALIDAD DE VIDA Y ESTADO DE SALUD EN PERSONAS MAYORES DE 60 AÑOS CON DEMENCIA INSTITUCIONALIZADAS (*)}

\author{
Carmen Rodríguez-Blázquez (1,2), Salomé Martín-García (3), Belén Frades-Payo (4), \\ María Soledad París (5), Iluminada Martínez-López (3) y Maria João Forjaz (6,7), en \\ nombre del Grupo Español de Investigación en Envejecimiento y Calidad de Vida.
}

(1) Centro Nacional de Epidemiología. Instituto de Salud Carlos III. Madrid.

(2) Centro de Investigación Biomédica en Enfermedades Neurodegenerativas. Madrid

(3) EULEN Servicios Socio-sanitarios. Madrid.

(4) Unidad de Investigación del Proyecto Alzheimer. Fundación CIEN. Fundación Reina Sofía. Instituto de Salud Carlos III. Madrid.

(5) Hospital Universitario Severo Ochoa. Leganés. Madrid.

(6) Escuela Nacional de Sanidad. Instituto de Salud Carlos III. Madrid.

(7) Red de Investigación en Servicios de Salud en Enfermedades Crónicas (REDISSEC).

(*) El estudio fue financiado por una ayuda de investigación de la Fundación CIEN (PI 017/09).

\section{RESUMEN}

Fundamentos: Es necesario profundizar en los distintos aspectos que componen la calidad de vida (CdV) en las personas con demencia que viven en residencias. El objetivo de este estudio fue describir la $\mathrm{CdV}$ e identificar los factores relacionados con ella.

Métodos: Muestra compuesta por 525 personas con demencia mayores de 60 años en 14 residencias de España. La CdV se evaluó mediante el EQ-5D (índice y escala visual analógica, EQ-EVA) y Quality of Life in Alzheimer's Disease (QoL-AD versiones residente y cuidador). También se utilizaron Clinical Dementia Rating Scale (CDR), el índice de Barthel, la escala Cornell de depresión en demencia (CSDD), una versión adaptada de la Cumulative Illness Rating Scale for Geriatrics (CIRS-G), Short Portable Mental Status Questionnaire (SPMSQ) y el Mini Examen Cognoscitivo (MEC). Para determinar la asociación entre variables se utilizó el coeficiente de correlación de Pearson y la prueba t de Student.

Resultados: Las escalas presentaron entre ellas correlaciones de 0,17 a 0,50. La puntuación QoL-AD cuidador fue mejor en varones con menos discapacidad y depresión $(28,94 \pm 4,91,29,91 \pm 4,74$ y $28,44 \pm 4,94$, respectivamente; $(\mathrm{p}<0,01)$ y correlacionó con el índice de Barthel 0,45 y con la CSDD -0,36. El QoL-AD residente fue mejor en ausencia de depresión $(29,29 \pm 6,03)$. El índice EQ-5D fue mayor en varones $(0,19 \pm 0,33)$ con menos discapacidad $(0,42 \pm 0,32)$ y su coeficiente de correlación con el índice de Barthel fue de 0,79 .

Conclusiones: En personas mayores con demencia institucionalizadas en residencias la calidad de vida se relaciona con el estado funcional y la depresión.

Palabras clave: Ancianos. Depresión. Personas con discapacidad. Demencia. Institucionalización. Calidad de vida.

Correspondencia

Carmen Rodríguez Blázquez

Centro Nacional de Epidemiología

Instituto de Salud Carlos III

Av. Monforte de Lemos, 5

28029 Madrid

crodb@isciii.es

\section{ABSTRACT}

\section{Quality of Life and Health Status} in Institutionalized Elderly with Dementian

Background: Studies that deepen in the aspects related to quality of life (QoL) of elderly with dementia living in nursing homes in Spain are needed. The aim of this study is to describe the QoL and related aspects in this population.

Methods: Sample of 525 people with dementia older than 60 years in 14 nursing homes. QoL was assessed with EQ-5D (both index and visual analogue Scale, EQ-VAS) and Quality of Life in Alzheimer's Disease(QoL-AD, resident and caregiver versions). Other scales were also applied: Clinical Dementia Rating Scale (CDR), Barthel Index, Cornell Scale for Depression in Dementia (CSDD), modified version of the Cumulative Illness Rating Scale for Geriatrics (CIRS-G), Short Portable Mental Status Questionnaire (SPMSQ) and Cognitive Mini-exam (MEC). To determine the relationship between the variables of interest, Pearson's correlation coefficient and the analysis of variance (Student's t test) were used.

Results: QoL scales displayed correlations from 0.17 to 0.50 between them. Qol-AD-caregiver scored higher in men with lower disability and depression $28.94 \pm 4.91,29.91 \pm 4.74$ and $28.44 \pm 4.94$, respectively; $(\mathrm{p}<0.01)$, and correlated 0.45 with Barthel Index and -0.36 with CSDD. Qol-ADresident scored higher in absence of depression (29.29 16.03$)$. EQ-5D Index scored higher in men $(0.19 \pm 0.33)$ with less disability $(0.42 \pm 0.32)$ and its coefficient of correlation with Barthel Index was 0.79 .

Conclusions: Functional state and depression are associated with quality of life in older people with dementia living in nursing homes.

Keywords: Dementia. Institutionalization. Quality of life. Aged. Depression. Disabled persons. 


\section{INTRODUCCIÓN}

La demencia es un síndrome asociado al envejecimiento. Debido a su alta prevalencia, a la necesidad de cuidados y de recursos que conlleva y a la inexistencia de tratamiento curativo en este momento, es importante profundizar en los aspectos que mejoran la calidad de vida (CdV) de las personas que las padecen. Según la Organización Mundial de la Salud (OMS), la calidad de vida es "la percepción que un individuo tiene de su lugar en la existencia, en el contexto de la cultura y del sistema de valores en los que vive y en relación con sus objetivos, sus expectativas, sus normas, sus inquietudes". Se trata de un concepto muy amplio que está influido de modo complejo por la salud física del sujeto, su estado psicológico, su nivel de independencia, sus relaciones sociales, así como su relación con los elementos esenciales de su entorno ${ }^{1}$.

En enfermedades crónicas tendentes a la dependencia, como es la demencia, la promoción del bienestar y el mantenimiento de una buena $\mathrm{CdV}$ es fundamental. Pese a los esfuerzos que se llevan haciendo en la última década, todavía quedan importantes interrogantes sobre este tema, especialmente en las personas mayores que viven en una residencia. En este sentido, la definición de las variables relacionadas con la $\mathrm{CdV}$ de las personas con demencia sigue siendo un reto, ya que existe gran variabilidad entre los aspectos relevantes en la $\mathrm{CdV}$ autopercibida y en la referida por sus cuidadores y el personal sanitario. Aspectos como la depresión, la función cognitiva o la autonomía inciden de modo distinto en la $\mathrm{CdV}$ cuando es referida por el propio paciente o bien por sus cuidadores o personal sanitario ${ }^{2}$.

Aproximadamente tres cuartas partes de las personas que viven en residencias padecen algún tipo de demencia ${ }^{3}$.

Conociendo los aspectos que más influyen en la $\mathrm{CdV}$, tanto físicos como emocionales e incluso ambientales ${ }^{4-6}$, podremos actuar sobre ellos de forma prioritaria y conseguir uno de los objetivos esenciales de la intervención, que las personas mayores con demencia tengan la mejor $\mathrm{CdV}$ posible. Es tan importante considerar este aspecto en el estudio de la demencia que incluso el Internacional Working Group for the Harmonization of Demencia Guidelines ${ }^{7}$ recomienda que la $\mathrm{CdV}$ sea incluida como un resultado a medir en los ensayos clínicos en sujetos con demencia.

El objetivo de este estudio fue describir la calidad de vida de personas mayores con demencia que viven en residencias de mayores así como el estudio de variables de salud asociadas.

\section{SUJETOS Y MÉTODOS}

Sujetos y procedimiento. El estudio se realizó en 14 centros de 10 provincias españolas, con una muestra de conveniencia de 525 personas institucionalizadas, mayores de 60 años y diagnosticadas de demencia según criterios del DSM-IV-TR ${ }^{8}$. Participaron diversos profesionales tanto a nivel sanitario (médicos, personal de enfermería, fisioterapeutas, terapeutas y auxiliares geriátricos) como psicosocial (psicólogos, trabajadores sociales, educadores y sociales), coordinados por los directores de los centros.

En todos los centros se realizó una reunión previa con el equipo multidisciplinar para analizar el cuestionario y la posterior distribución de escalas por departamentos, adecuando el tipo de escala al perfil profesional.

El estudio fue aprobado por el Comité de Ética de la Investigación y de Bienestar Animal (CEIyBA) del Instituto de Salud Carlos III. Los participantes o sus representantes legales firmaron el consentimiento informado.

Se registraron las variables sociodemográficas de los residentes (sexo, edad, nivel de estudios y estado civil), participación en 
actividades de ocio y tiempo libre y tipo de demencia (según la historia clínica). Para la evaluación de la independencia funcional se utilizó el índice de Barthel, que valora 10 actividades básicas de la vida cotidiana, con una puntuación total entre 0 y $100^{9,10}$. Este índice es ampliamente usado para valorar el grado de discapacidad al ingreso en residencias asistidas y en nuestro estudio fue aplicado por el personal médico.

Las condiciones médicas crónicas se estimaron con una escala de comorbilidad basada en el Cumulative Illness Rating Scale for Geriatrics (CIRS-G) ${ }^{11}$, un instrumento específico para personas mayores que posee buenas propiedades clinimétricas. En el presente estudio se recogió a partir de la historia clínica el número de condiciones crónicas que presentaba el sujeto sin valorar su gravedad.

Con el fin de obtener una medida genérica y estandarizada de la $\mathrm{CdV}$ relacionada con la salud y estado de salud se utilizó el EQ-5D ${ }^{12-14}$, que consta de dos partes. La primera evalúa cinco dimensiones: movilidad, cuidado personal, actividades cotidianas, dolor/malestar y ansiedad/depresión. Las respuestas a las 5 dimensiones en tres niveles generan un perfil que, mediante una fórmula, se transforma en un índice (índice EQ-5D) que puntúa entre -1 y 1, en el que el 1 indica la mejor CdV. La segunda parte es una escala visual analógica (EQ-VAS) que evalúa el estado de salud actual en una escala de 0 a 100. El EQ-5D, en su versión por informante, fue contestado por un cuidador profesional sanitario o por un familiar en relación al paciente.

Como instrumento de evaluación de la $\mathrm{CdV}$ específico para población con demencia se aplicó la escala QoL-AD ${ }^{15-17}$, en las versiones del residente (por entrevista) y del cuidador. Ambas cuentan con 13 ítems que se puntúan en una escala de 1 (malo) a 4 (excelente). La puntuación total máxima es de 52 puntos. El QoL-AD en general presenta buenas propiedades psicométricas. La versión por entrevista incluye instrucciones para el entrevistador. El estado cognitivo de algunos sujetos hizo que no entendieran las preguntas y por ello no se les pudo aplicar directamente el QoL-AD.

El estado cognitivo se valoró con dos instrumentos de cribado. El cuestionario Short Portable Mental Status Questionnaire $(\mathrm{SPMSQ})^{18}$ que consta de 10 ítems (una puntuación de 3 o más indica deterioro cognitivo) muy utilizado en atención primaria. Está validado en España ${ }^{19}$ y presenta buenas propiedades psicométricas. Por otra parte, se aplicó también el Mini-Examen Cognoscitivo $(\mathrm{MEC})^{20}$, un cuestionario breve de evaluación de las capacidades cognitivas. Para este estudio se utilizó la versión de 35 puntos, en la que los ítems están agrupados en 5 apartados: orientación, memoria de fijación, concentración y cálculo, recuerdo diferido y lenguaje y construcción. La puntuación oscila entre 0 y 35 , con un punto de corte de 23/24 indicativo de deterioro cognitivo en pacientes geriátricos ${ }^{19}$. Estas escalas fueran aplicadas por el personal clínico de las residencias.

Como medida global del grado de demencia, se utilizó el Clinical Dementia Rating $(\mathrm{CDR})^{21}$ que evalúa seis dominios cognitivos y funcionales y que fue aplicada por el personal clínico de las residencias. La puntuación de cada dominio oscila entre 0 (sin demencia) y 3 (demencia grave). La puntuación global se obtiene a partir de un algoritmo $^{22}$. Es una escala ampliamente utilizada en clínica e investigación, validada en español y con datos normativos y psicométricos muy aceptables.

Para evaluar los síntomas depresivos se utilizó la Cornell Scale for Depression in Dementia (CSDD) ${ }^{23,24}$, específicamente diseñada para pacientes con deterioro cognitivo y que fue aplicada por el personal clínico de las residencias. La CSDD tiene 19 ítems, con una puntuación total máxima de 38 , y un punto de corte de 7/8 para sintomatología depresiva significativa ${ }^{25-27}$. 
Análisis estadístico. Se usaron estadísticos descriptivos para los datos sociodemográficos y las variables de resultados de las escalas (frecuencia, media y desviación estándar). A través de la prueba de Kolmogorov-Smirnov se comprobó si las puntuaciones de las escalas MEC, EQ-5D (índice y EVA), QoL-AD (cuidador y residente) y número de enfermedades seguían una distribución normal.

Se consideraron como variables dependientes el QoL-AD contestado por el residente y cuidador y el índice EQ-5D. Para analizar la asociación entre variables de interés se utilizó el coeficiente de correlación de Pearson. Los valores $\geq 0,60$ fueron considerados altos, de 0,40 a0,59 moderados y de 0,20 a 0,39 débiles $^{28}$. Además, la muestra se dividió en grupos por las siguientes variables: sexo, edad (60-85 y 86 y más), nivel educativo ( $\sin$ estudios o primarios incompletos versus con estudios primarios o más), por estado civil, por número de problemas de salud crónicos (según la mediana: $\leq 7 \mathrm{y}>7$ ) y por el punto de corte de la CSDD $(7 / 8)^{25-27}$. Se analizó si las puntuaciones de las escalas EQ-5D (índice y EVA) y QoL-AD presentaban diferencias significativas en función de los grupos anteriormente descritos mediante tests t de Student.

\section{RESULTADOS}

La muestra estuvo formada por 525 residentes con una edad media de 85,57 años (desviación típica: $\mathrm{DT}=6,73$, rango: $60-102$ ), de los que $434(82,87 \%)$ eran mujeres (tabla 1). El $62,64 \%$ eran viudos y el $65,8 \%$ tenían un nivel de estudios inferior a primaria. El $44,4 \%$ de los residentes practicaban un tipo de ocio pasivo (ver la televisión, escuchar música, etcétera). El 36,4\% tenía demencia tipo Alzheimer, aunque hay que destacar que para el $36,2 \%$ el diagnóstico no estaba especificado.

En la tabla 2 se muestran los resultados de las escalas de evaluación utilizadas. El 57,9\% de la muestra presentó discapacidad grave o total y el $61,5 \%$ tenía un nivel de demencia grave según el CDR, predominando los residentes con enfermedad de Alzheimer (36,4\%). Por último el 44,2\% de la muestra tenía sintomatología depresiva según el punto de corte de la CSDD. La tasa de respuesta para el QoLAD alcanzó el $60 \%$ cuando fue contestado por el cuidador debido a los casos perdidos en un ítem (matrimonio/relación personal estrecha) y el $28 \%$ cuando fue contestado por el residente. No se observaron diferencias estadísticamente significativas en las puntuaciones del QoL-AD en los 124 casos en que fue contestado tanto por el cuidador como por el residente (test $t$ de Student para muestras pareadas). Sin embargo, en los 366 casos en los que sólo respondió el cuidador al QoL-AD se detectaron diferencias estadísticamente significativas en sexo, CDR, índice de Barthel, CSDD, MEC y EQ-5D (índice y EVA) con el grupo de 124 residentes que sí lo respondieron (pruebas t de Student y Mann-Whitney, $\mathrm{p}<0,001$ ), observándose peores indicadores de salud y mayor proporción de mujeres en el primer grupo $(85,12 \%$ frente a $71,77 \%$ ).

La tabla 3 presenta los coeficientes de correlación de las escalas de $\mathrm{CdV}$ con el resto de medidas utilizadas. Las escalas de CdV se asociaron significativamente entre sí, con correlaciones entre 0,17 (QoL-AD residente e índice EQ-5D) y 0,50 (QoL-AD-cuidador con EQ-5D índice y EVA). La correlación entre el QoL-AD contestado por el residente y el cuidador fue de $(0,38)$. El coeficiente de correlación de la escala QoL-AD-cuidador con el índice de Barthel fue de 0,48 mientras que con la CSDD fue de -0,36. La correlación de la escala QoL-AD-residente con el resto de escalas fue nula o débil, excepto con la EQ-EVA $(\mathrm{r}=0,33)$. El coeficiente de correlación entre el índice EQ-5D y el índice de Barthel fue de 0,79 .

El análisis de las puntuaciones de las escalas de $\mathrm{CdV}$ en función de variables de interés aparece en la tabla 4. La puntuación de la escala QoL-AD-cuidador fue mejor en hombres $(28,94 \pm 4,91)$ que en mujeres $(27,19 \pm 5,24)$, en 


\begin{tabular}{|c|c|}
\hline \multicolumn{2}{|c|}{$\begin{array}{c}\text { Tabla } 1 \\
\text { Características } \\
\text { de las personas de la muestra }\end{array}$} \\
\hline Variables & n (\%) \\
\hline Sexo (mujer) & $434(82,7)$ \\
\hline Edad: 86 y más (vs 60-85) & $277(52,8)$ \\
\hline $\begin{array}{l}\text { Estado civil } \\
\text { Soltero } \\
\text { Casado/En pareja } \\
\text { Separado } \\
\text { Viudo }\end{array}$ & $\begin{array}{r}85(16,3) \\
98(18,8) \\
12(2,3) \\
327(62,6)\end{array}$ \\
\hline $\begin{array}{r}\text { Nivel de estudios } \\
\text { Sin estudios/Primaria } \\
\text { incompleta } \\
\text { Primaria } \\
\text { Secundaria/Universitarios }\end{array}$ & $\begin{array}{r}137(26,3) \\
41(7,9)\end{array}$ \\
\hline $\begin{array}{l}\text { Historial laboral } \\
\text { Trabajó antes (vs. no } \\
\text { trabajó nunca) }\end{array}$ & $272(54,3)$ \\
\hline $\begin{array}{r}\text { Actividades de ocio } \\
\text { Ocio activo } \\
\text { Ocio pasivo } \\
\text { Ocio cultural } \\
\text { Ocio social } \\
\text { Otras actividades (ej: taller } \\
\text { cognitivo) }\end{array}$ & $\begin{array}{r}185(35,2) \\
233(44,4) \\
95(18,1) \\
144(27,4) \\
31(5,9)\end{array}$ \\
\hline $\begin{array}{r}\text { Tipo de demencia } \\
\text { Alzheimer } \\
\text { vascular } \\
\text { mixta } \\
\text { inespecífica } \\
\text { Otra } \\
\text { Sin diagnóstico diferencial }\end{array}$ & $\begin{array}{r}191(36,4) \\
34(6,5) \\
23(4,4) \\
71(13,5) \\
16(3,0) \\
190(36,2)\end{array}$ \\
\hline
\end{tabular}

${ }^{*}$ El SPSMQ no pudo aplicarse en 214 sujetos. SPSMQ: Short Portable Mental Status Questionnaire; CDR: Clinical Dementia Rating; CSDD: Escala de Depresión de Cornell; DT: desviación típica; EVA: escala visual analógica.

\begin{tabular}{|c|c|c|}
\hline \multicolumn{3}{|c|}{$\begin{array}{c}\text { Tabla } 2 \\
\text { Estadísticas descriptivas } \\
\text { de las escalas utilizadas }\end{array}$} \\
\hline Escalas & n ( & \\
\hline $\begin{array}{r}\text { Grado de discapacidad } \\
\text { (según índice de Barthel) } \\
\text { Grave o total } \\
\text { Moderada } \\
\text { Leve } \\
\text { Sin discapacidad o mínima }\end{array}$ & \multicolumn{2}{|c|}{$\begin{array}{r}304(57,9) \\
91(17,3) \\
61(11,6) \\
56(10,7)\end{array}$} \\
\hline $\begin{array}{l}\text { SPMSQ } \\
\quad \text { Positivo (deterioro cognitivo) }\end{array}$ & \multicolumn{2}{|c|}{$288(93,2)^{*}$} \\
\hline $\begin{array}{r}\text { Gravedad de la demencia }(\mathrm{CDR}) \\
\text { Demencia grave }(\mathrm{CDR}=3) \\
\text { Demencia moderada }(\mathrm{CDR}=2) \\
\text { Demencia leve }(\mathrm{CDR}=1)\end{array}$ & \multicolumn{2}{|c|}{$\begin{array}{r}323(61,5) \\
130(24,8) \\
72(13,7)\end{array}$} \\
\hline $\begin{array}{l}\text { Sintomatología depresiva } \\
\text { Sí ( } \geq 8 \text { puntos en CSDD) }\end{array}$ & \multicolumn{2}{|c|}{$123(44,2)$} \\
\hline Escalas & Media $\pm \mathrm{DT}$ & Rango \\
\hline Índice de Barthel $(\mathrm{n}=512)$ & $32,8 \pm 29,5$ & $0-100$ \\
\hline $\begin{array}{l}N^{o} \text { de condiciones médicas cróni- } \\
\text { cas }(n=511)\end{array}$ & $7,8 \pm 2,8$ & $2-15$ \\
\hline $\begin{array}{l}\text { Escala de Depresión de Cornell } \\
(\mathrm{n}=278)\end{array}$ & $7,4 \pm 6,1$ & $0-29$ \\
\hline $\begin{array}{l}\text { Mini-Examen Cognoscitivo } \\
(\mathrm{MEC})(\mathrm{n}=319)\end{array}$ & $13,2 \pm 8,1$ & $0-33$ \\
\hline Índice EQ-5D $(\mathrm{n}=514)$ & $0,11 \pm 0,4$ & $-0,65-1$ \\
\hline EQ-5D EVA $(n=510)$ & $51,5 \pm 21,5$ & $0-100$ \\
\hline QOL-AD residente $(\mathrm{n}=148)$ & $31,4 \pm 6,8$ & $13-51$ \\
\hline QOL-AD cuidador $(n=366)$ & $27,5 \pm 5,2$ & $13-41$ \\
\hline
\end{tabular}

El índice de Barthel, número de condiciones médicas crónicas, Escala de Depresión de Cornell y MEC fueron respondidas por personal sanitario de las residencias. El EQ-5D (índice y EVA) fue respondido por el cuidador (profesional o familiar).

\begin{tabular}{|c|c|c|c|}
\hline \multicolumn{4}{|c|}{$\begin{array}{c}\text { Tabla } 3 \\
\begin{array}{c}\text { Coeficientes de correlación entre las escalas de calidad de vida y las de salud } \\
\text { utilizadas en el estudio }\end{array}\end{array}$} \\
\hline & QoL-AD cuidador & QoL-AD residente & Índice EQ-5D \\
\hline QOL-AD residente & 0,38 & & \\
\hline Indice EQ-5D & 0,50 & 0,17 & \\
\hline EQ-EVA & 0,50 & 0,33 & 0,58 \\
\hline Índice de Barthel & 0,48 & $0,12^{*}$ & 0,79 \\
\hline $\mathrm{N}^{\circ}$ de condiciones médicas crónicas & $-0,09^{*}$ & $-0,22$ & $-0,11$ \\
\hline Escala de Depresión de Cornell & $-0,36$ & $-0,25$ & $-0,27$ \\
\hline Mini-Examen Cognoscitivo (MEC) & 0,31 & $-0,18$ & 0,39 \\
\hline SPSMQ & $-0,04^{*}$ & 0,17 & $-0,28$ \\
\hline Clinical Dementia Rating (CDR) & $-0,38$ & $0,01^{*}$ & $-0,51$ \\
\hline
\end{tabular}




\begin{tabular}{|c|c|c|c|}
\hline \multicolumn{4}{|c|}{$\begin{array}{c}\text { Tabla } 4 \\
\text { Medias y desviaciones típicas de las escalas de calidad de vida } \\
\text { en función de variables de interés (prueba t de Student) }\end{array}$} \\
\hline & $\begin{array}{l}\text { QoL-AD cuidador } \\
\quad(\mathrm{n}=366)\end{array}$ & $\begin{array}{l}\text { QoL-AD residente } \\
(\mathrm{n}=148)\end{array}$ & $\begin{array}{l}\text { Índice EQ-5D } \\
(\mathrm{N}=514)\end{array}$ \\
\hline $\begin{array}{r}\text { Hombre } \\
\text { Mujer }\end{array}$ & $\begin{array}{c}\mathrm{p}=0,01 \\
28,94 \pm 4,91 \\
27,19 \pm 5,24\end{array}$ & $\begin{array}{c}\text { no significativo } \\
30,24 \pm 6,17 \\
31,82 \pm 7,09\end{array}$ & $\begin{array}{c}\mathrm{p}=0,03 \\
0,19 \pm 0,33 \\
0,10 \pm 0,39\end{array}$ \\
\hline $\begin{array}{r}\text { 60-85 años } \\
86 \text { años y más }\end{array}$ & $\begin{array}{c}\text { no significativo } \\
27,65 \pm 5,28 \\
27,42 \pm 5,17\end{array}$ & $\begin{array}{c}\text { no significativo } \\
31,24 \pm 7,26 \\
31,53 \pm 6,37\end{array}$ & $\begin{array}{c}\mathrm{p}=0,04 \\
0,15 \pm 0,40 \\
0,08 \pm 0,37\end{array}$ \\
\hline $\begin{array}{l}\text { Nivel de estudios: } \\
\text { Sin estudios o < primarios } \\
\text { Primarios o superior }\end{array}$ & $\begin{array}{c}\mathrm{p}=0,001 \\
26,78 \pm 5,15 \\
28,71 \pm 5,12\end{array}$ & $\begin{array}{c}\text { no significativo } \\
30,76 \pm 6,64 \\
32,44 \pm 7,04\end{array}$ & $\begin{array}{c}\text { no significativo } \\
0,11 \pm 0,40 \\
0,12 \pm 0,35\end{array}$ \\
\hline $\begin{array}{r}\text { Sin pareja } \\
\text { Con pareja }\end{array}$ & $\begin{array}{c}\text { no significativo } \\
27,37 \pm 5,35 \\
27,87 \pm 4,82 \\
\end{array}$ & $\begin{array}{c}\text { no significativo } \\
31,33 \pm 6,65 \\
31,69 \pm 7,51 \\
\end{array}$ & $\begin{array}{c}\text { no significativo } \\
0,12 \pm 0,40 \\
0,10 \pm 0,34 \\
\end{array}$ \\
\hline $\begin{array}{r}\text { Número de problemas crónicos: } \\
7 \text { o menos } \\
\text { Más de } 7\end{array}$ & $\begin{array}{c}\text { no significativo } \\
27,30 \pm 5,39 \\
27,16 \pm 4,94\end{array}$ & $\begin{array}{c}\text { no significativo } \\
31,67 \pm 7,05 \\
31,05 \pm 6,62\end{array}$ & $\begin{array}{c}\text { no significativo } \\
0,14 \pm 0,38 \\
0,08 \pm 0,39\end{array}$ \\
\hline $\begin{array}{r}\text { Discapacidad (Índice de Barthel): } \\
\text { Grave o total } \\
\text { Leve/moderada o sin discapacidad }\end{array}$ & $\begin{array}{c}\mathrm{p}<0,001 \\
25,73 \pm 4,89 \\
29,91 \pm 4,74\end{array}$ & $\begin{array}{c}\text { no significativo } \\
30,22 \pm 7,10 \\
31,65 \pm 6,28\end{array}$ & $\begin{array}{c}\mathrm{p}<0,001 \\
-0,11 \pm 0,25 \\
0,42 \pm 0,32\end{array}$ \\
\hline $\begin{array}{r}\text { Depresión (escala de Cornell): } \\
\text { Hasta } 7 \text { puntos } \\
8 \text { puntos o más }\end{array}$ & $\begin{array}{c}\mathrm{p}=0,03 \\
28,44 \pm 4,94 \\
27,19 \pm 5,25\end{array}$ & $\begin{array}{c}\mathrm{p}=0,007 \\
32,54 \pm 7,09 \\
29,29 \pm 6,03\end{array}$ & $\begin{array}{c}\text { no significativo } \\
0,15 \pm 0,39 \\
0,10 \pm 0,37 \\
\end{array}$ \\
\hline
\end{tabular}

residentes con estudios primarios o superiores $(28,71 \pm 5,12$ frente a $26,78 \pm 5,15$ en residentes con un nivel inferior de estudios) y en aquellos con menor grado de discapacidad $(29,91 \pm 4,74$ frente a 25,73 $\pm 4,89$ de los residentes con discapacidad grave o total) y de depresión $(28,44 \pm 4,94$ en residentes sin depresión, frente a $27,19 \pm 5,25$ en los residentes con depresión), siendo las diferencias estadísticamente significativa $(\mathrm{p}<0,01)$, mientras que la versión del residente obtuvo puntuaciones más altas en los residentes con menor puntuación en la escala de depresión $(32,54 \pm 7,09$, frente a $29,29 \pm 6,03$ en los residentes con posible depresión), diferencias que también fueron estadíticamente significativas $(\mathrm{p}=0,007)$. El índice EQ-5D fue más alto en hombres $(0,19 \pm 0,33)$ que en mujeres $(0,10 \pm 0,39)$, en el grupo de residentes de menor edad $(0,15 \pm 0,40$ en los residentes de hasta 85 años, frente a $0,08 \pm 0,37$ en los residentes de 86 años y más) y con menor grado de discapacidad $(0,42 \pm 0,32$, frente a $-0,11 \pm 0,25$ en los residentes con discapacidad grave y total) $(\mathrm{p}<0,04)$.

\section{DISCUSIÓN}

Los principales resultados de este estudio indican que la $\mathrm{CdV}$ de las personas con demencia está relacionada con su nivel de funcionamiento y con la presencia de síntomas depresivos cuando la CdV está valorada por el cuidador y por la sintomatología depresiva cuando es el propio residente con demencia quien evalúa su $\mathrm{CdV}$.

Este estudio profundiza en todos aquellos aspectos referentes a la $\mathrm{CdV}$ de las personas con demencia institucionalizadas y arroja luz sobre las variables que pueden ser relevantes para la definición del concepto contribuyendo así al establecimiento de prioridades en la intervención sanitaria de esta población desde una perspectiva holística.

El uso de diferentes escalas y perspectivas es recomendable debido a las dificultades que conlleva evaluar la $\mathrm{CdV}$ en personas con demencia, que pueden presentar pérdidas de memoria y problemas para com- 
prender preguntas, expresar ideas y juicios sobre uno mismo, etc. ${ }^{2,14,15}$. Se observó que las distintas medidas de CdV se asocian más entre sí que el mismo cuestionario cuando es valorado por distintos informantes.

Las puntuaciones de $\mathrm{CdV}$ informadas por el cuidador estuvieron afectadas por la mayor discapacidad (índice de Barthel) y el peor estado de salud (EQ-EVA) de los residentes, de una forma más intensa que en las puntuaciones de la $\mathrm{CdV}$ del residente. $\mathrm{La}$ discapacidad o el nivel de funcionamiento es un componente central de $1 \mathrm{a} \mathrm{CdV}^{29}$ y en distintos estudios se identifica como uno de los determinantes principales de la $\mathrm{CdV}$ global en personas mayores ${ }^{30}$. Los modelos de $\mathrm{CdV}$ en esta población y en personas con demencia relacionan la $\mathrm{CdV}$ global con el nivel de funcionamiento de manera directa ${ }^{5,31,32}$. La presencia de dependencia funcional también se ha revelado como un factor determinante en la percepción del cuidador sobre la $\mathrm{CdV}$ de la persona con demencia ${ }^{2,33}$. Esto también podría explicar el hecho de que en nuestro estudio las escalas de $\mathrm{CdV}$ respondidas por el cuidador estén asociadas a discapacidad pero no el QoL-AD-residente.

La depresión influye negativamente en la evaluación de la CdV medida por la escala QoL-AD tanto autoinformada como notificada por el cuidador. Es un hecho conocido que la depresión es un importante determinante en la $\mathrm{CdV}$ global de las personas con demencia ${ }^{2,34}$, bien influyendo directamente en la misma o bien mediando en la relación entre estado de salud y $\mathrm{CdV}^{30}$. Sin embargo, en general, los coeficientes de correlación entre las medidas de CdV y la CSDD son de bajos a moderados, un resultado ya descrito anteriormente en un grupo de personas con demencia grave ${ }^{35}$, probablemente debido a que la mayoría de los individuos de nuestro estudio no padecen depresión.

En nuestra investigación no se evidenció relación entre la $\mathrm{CdV}$ y el estado cognitivo o la gravedad de la demencia, exceptuando en el caso de la correlación entre EQ-5D y
CDR. Distintos estudios indican que los cuidadores tienden a infravalorar la $\mathrm{CdV}$ de los sujetos con demencia mientras que los pacientes tienden a dar mejores puntuaciones en $\mathrm{CdV}$, especialmente en estadios avanzados de la enfermedad, por lo que la relación entre dichas variables no es directa ${ }^{35-39}$.

Como limitaciones del estudio es necesario indicar que no se pueden extraer conclusiones sobre causalidad ni sus resultados pueden ser generalizados al conjunto de personas mayores con demencia institucionalizadas, por haber contado con una muestra de conveniencia no aleatoria y tener un diseño transversal. No obstante, el tamaño muestral, sus características socio-demográficas y clínicas así como su distribución geográfica son suficientemente amplios como para garantizar la validez externa. La composición de la muestra resultó ser mayoritariamente femenina y con una edad superior a los 80 años, con un grado de dependencia funcional y de demencia entre moderado y grave, al igual que en artículos previos ${ }^{38,40}$. Por tanto, a pesar de que la selección no fue aleatoria, las características de la población son similares a la de estudios previos, lo que permite la comparación con ellos. Por último, hay que destacar que algunas escalas presentan un gran número de datos perdidos, ya que fue imposible administrarlas a determinados sujetos por su incapacidad para contestar. Sin embargo, no contar con los datos de este grupo haría que se perdiera información muy importante, especialmente de las personas con demencia más avanzada. Esto hace aún más trascendental la información que puede aportar el cuidador en relación a la persona con demencia ${ }^{41,42}$.

Lo más relevante del presente estudio es el uso de distintas escalas de CdV y perspectivas (cuidador y residente), lo cual permite profundizar en la descripción de la $\mathrm{CdV}$ y el estado de salud de los mayores con demencia. Es importante, por tanto, tener en cuenta ambas perspectivas a la hora de valorar el estado de salud y la $\mathrm{CdV}$ de 
personas con demencia. En el presente estudio se evidencia que mientras que el estado funcional y la sintomatología depresiva son relevantes para la evaluación de la $\mathrm{CdV}$ por parte del cuidador, la valoración del propio residente se relaciona con los síntomas de depresión. Es imprescindible insistir en la importancia de la identificación de problemas en los aspectos funcionales y emocionales de las personas con demencia así como la relación entre estas variables y la $\mathrm{CdV}$, mediante la realización de más estudios que identifiquen prioridades en la intervención, diseñen y propongan programas de intervención específicos y evalúen su efectividad.

\section{AGRADECIMIENTOS}

El Grupo Español de Investigación en Envejecimiento y Calidad de Vida está compuesto por: Alba Ayala, Maria João Forjaz, Pablo Martínez-Martín y Carmen Rodríguez-Blázquez, del Instituto de Salud Carlos III. Gloria Fernández-Mayoralas y Fermina Rojo-Pérez, del Consejo Superior de Investigaciones Científicas. Belén Frades-Payo, Beatriz León-Pérez y Marina Ávila, de la Unidad de Investigación del Proyecto Alzheimer, Fundación CIEN-Fundación Reina Sofía. Salomé Martín-García e Iluminada Martínez, de Eulen Servicios Sociosanitarios y María Eugenia Prieto-Flores, de la Universidad Nacional de Educación a Distancia.

Los autores agradecen al personal de las residencias participantes en el estudio por su valiosa colaboración en el mismo.

\section{BIBLIOGRAFÍA}

1. The World Health Organization Quality of Life assessment (WHOQOL): position paper from the World Health Organization. Soc Sci Med. 1995;41(10):14039.

2. Crespo M, Hornillos C, de Quirós MB. Factors associated with quality of life in dementia patients in longterm care. Int Psychogeriatr. 2013;25(4):577-85.

3. Hoe J, Hancock G, Livingston G, Orrell M. Quality of life of people with dementia in residential care homes. Br J Psychiatry. 2006;188:460-4.
4. Garre-Olmo J, López-Pousa S, Turon-Estrada A, Juvinyà D, Ballester D, Vilalta-Franch J. Environmental determinants of quality of life in nursing home residents with severe dementia. J Am Geriatr Soc. 2012;60(7):1230-6.

5. León-Salas B, Olazarán J, Cruz-Orduña I, AgüeraOrtiz L, Dobato JL, Valentí-Soler M, et al. Quality of life (QoL) in community-dwelling and institutionalized Alzheimer's disease (AD) patients. Arch Gerontol Geriatr. 2013;57(3):257-62.

6. Castro-Monteiro E, Forjaz MJ, Ayala A, Rodriguez-Blazquez C, Fernandez-Mayoralas G, DiazRedondo A, et al. Change and predictors of quality of life in institutionalized older adults with dementia. Qual Life Res. 2014;23(9):2595-601.

7. Mack JL, Whitehouse PJ. Quality of Life in Dementia: state of the art--report of the International Working Group for Harmonization of Dementia Drug Guidelines and the Alzheimer's Society satellite meeting. Alzheimer Dis Assoc Disord. 2001;15(2):69-71.

8. American Psychiatric Association. Diagnostical and Statistical Manual of Mental Disorders: DSMIV-TR: 4th Edition, Text Revision. Washington DC: American Psychiatric Association; 2000.

9. Mahoney FI, Barthel DW. Functional evaluation: the Barthel Index. Md State Med J. 1965;14:61-5.

10. Baztán JJ, Pérez del Molino J, Alarcón T, San Cristobal E, Izquierdo G, Manzarbeitia I. Indice de Barthel: Instrumento válido para la valoración funcional de pacientes con enfermedad cerebrovascular. Rev Esp Geriatr Gerontol. 1993;28:32-40.

11. Miller MD, Paradis CF, Houck PR, Mazumdar S, Stack JA, Rifai AH, et al. Rating chronic medical illness burden in geropsychiatric practice and research: application of the Cumulative Illness Rating Scale. Psychiatr Res. 1992;41(3):237-48.

12. EuroQol Group. EuroQol--a new facility for the measurement of health-related quality of life. The EuroQol Group. Health Policy. 1990;16(3):199-208.

13. Badia X, Roset M, Montserrat S, Herdman M, Segura A. La versión española del EuroQol: descripción y aplicaciones. Med Clín. 1999;112 Suppl 1:79-85.

14. Diaz-Redondo A, Rodriguez-Blazquez C, Ayala A, Martinez-Martin P, Forjaz MJ, Spanish Research Group on Quality of Life and Aging. EQ-5D rated by proxy in institutionalized older adults with dementia: psychometric pros and cons. Geriatr Gerontol Int. 2014;14(2):346-53.

15. Logsdon RG, Gibbons LE, McCurry SM, Teri L. Assessing quality of life in older adults with cognitive impairment. Psychosom Med. 2002;64(3):510-9. 
16. Gómez-Gallego M, Gómez-Amor J, Gómez-García J. Validación de la versión española de la escala QoL-AD en pacientes con enfermedad de Alzheimer, cuidadores y profesionales sanitarios. Neurología. 2012;27(1):4-10.

17. León-Salas B, Logsdon RG, Olazarán J, MartínezMartín P, The Msu-Adru. Psychometric properties of the Spanish QoL-AD with institutionalized dementia patients and their family caregivers in Spain. Aging Ment Health. 2011;15(6):775-83.

18. Pfeiffer E. A short portable mental status questionnaire for the assessment of organic brain deficit in elderly patients. J Am Geriatr Soc. 1975;23(10):433-41.

19. Martínez de la Iglesia J, Dueñas Herrero R, Onís Vilches MC, Aguado Taberné C, Albert Colomer C, Luque Luque R. Adaptación y validación al castellano del cuestionario de Pfeiffer (SPMSQ) para detectar la existencia de deterioro cognitivo en personas mayores de 65 anos. Med Clín. 2001;117(4):129-34.

20. Lobo A, Saz P, Marcos G, Día JL, de la Cámara C, Ventura T, et al. Revalidación y normalización del Mini-Examen Cognoscitivo (primera versión en castellano del Mini-Mental Status Examination) en la población general geriátrica. Med Clín. 1999;112(20):76774.

21. Hughes CP, Berg L, Danziger WL, Coben LA, Martin RL. A new clinical scale for the staging of dementia. Br J Psychiatry. 1982;140:566-72.

22. Morris JC. The Clinical Dementia Rating (CDR): current version and scoring rules. Neurology. 1993;43(11):2412-4.

23. Alexopoulos GS, Abrams RC, Young RC, Shamoian CA. Cornell Scale for Depression in Dementia. Biol Psychiatry. 1988;23(3):271-84.

24. Pujol J, De Azpiazu P, Salamero M, Cuevas R. Sintomatología depresiva de la demencia. Escala de Cornell: validación de la versión en castellano. Rev Neurol. 2001 Aug 16;33(4):397-8.

25. Vida S, Des Rosiers P, Carrier L, Gauthier S. Depression in Alzheimer's disease: receiver operating characteristic analysis of the Cornell Scale for Depression in Dementia and the Hamilton Depression Scale. J Geriatr Psychiatry Neurol. 1994 Sep;7(3):159-62.

26. Snowdon J, Fleming R. Recognising depression in residential facilities: an Australian challenge. Int J Geriatr Psychiatry. 2008 Mar;23(3):295-300.

27. Gerritsen DL, Smalbrugge M, Teerenstra S, Leontjevas R, Adang EM, Vernooij-Dassen MJFJ, et al. Act In case of Depression: the evaluation of a care program to improve the detection and treatment of depression in nursing homes. Study Protocol. BMC Psychiatry. 2011;11:91.
28. Fisk JD, Brown MG, Sketris IS, Metz LM, Murray TJ, Stadnyk KJ. A comparison of health utility measures for the evaluation of multiple sclerosis treatments. J Neurol Neurosurg Psychiatry. 2005;76(1):58-63.

29. Bowling A, Gabriel Z. An Integrational Model of Quality of Life in Older Age. Results from the ESRC/ MRC HSRC Quality of Life Survey in Britain. Soc Indic Res. 2004;69(1):1-36.

30. Bowling A, Banister D, Sutton S, Evans O, Windsor J. A multidimensional model of the quality of life in older age. Aging Ment Health. 2002;6(4):355-71.

31. Sullivan MD, Kempen GI, Van Sonderen E, Ormel J. Models of health-related quality of life in a population of community-dwelling Dutch elderly. Qual Life Res. 2000;9(7):801-10.

32. Byrne-Davis LMT, Bennett PD, Wilcock GK. How are quality of life ratings made? Toward a model of quality of life in people with dementia. Qual Life Res Int J Qual Life Asp Treat Care Rehabil. 2006 Jun;15(5):855-65.

33. Conde-Sala JL, Garre-Olmo J, Turró-Garriga O, López-Pousa S, Vilalta-Franch J. Factors related to perceived quality of life in patients with Alzheimer's disease: the patient's perception compared with that of caregivers. Int J Geriatr Psychiatry. 2009;24(6):58594.

34. Bosboom PR, Alfonso H, Eaton J, Almeida OP. Quality of life in Alzheimer's disease: different factors associated with complementary ratings by patients and family carers. Int Psychogeriatr. 2012;24(5):708-21.

35. Hoe J, Katona C, Roch B, Livingston G. Use of the QOL-AD for measuring quality of life in people with severe dementia--the LASER-AD study. Age Ageing. 2005;34(2):130-5.

36. Arlt S, Hornung J, Eichenlaub M, Jahn H, Bullinger M, Petersen $C$. The patient with dementia, the caregiver and the doctor: cognition, depression and quality of life from three perspectives. Int $\mathbf{J}$ Geriatr Psychiatry. 2008;23(6):604-10.

37. Waldorff FB, Nielsen ABS, Waldemar G. Self-rated health in patients with mild Alzheimer's disease: baseline data from the Danish Alzheimer Intervention Study. Arch Gerontol Geriatr. 2010;50(1):1-5.

38. Kunz S. Psychometric properties of the EQ-5D in a study of people with mild to moderate dementia. Qual Life Res. 2010;19(3):425-34.

39. Conde-Sala JL, Turró-Garriga O, Garre-Olmo J, Vilalta-Franch J, Lopez-Pousa S. Discrepancies regarding the quality of life of patients with Alzheimer's disease: a three-year longitudinal study. J Alzheimers Dis. 2014;39(3):511-25. 
40. Van Malderen L, Mets T, Gorus E. Interventions to enhance the Quality of Life of older people in residential long-term care: a systematic review. Ageing Res Rev. 2013;12(1):141-50.

41. Crespo M, Bernaldo de Quirós M, Gómez MM, Hornillos C. Quality of life of nursing home residents with dementia: a comparison of perspectives of residents, family, and staff. Gerontologist. 2012;52(1):5665.

42. Crespo M, Hornillos C, Gómez MM. Assessing quality of life of nursing home residents with dementia: feasibility and limitations in patients with severe cognitive impairment. Int Psychogeriatr. 2013;25(10):1687-95. 